\title{
A11
}

doi: 10.14232/ syrpharmacognosy.2021.a11

\section{Phytochemical and pharmacological study of furocoumarins in Citrus trifoliata L.}

\section{Diána Kerekes}

Email: kerekesdia@pharmacognosy.hu

Furocoumarins are heterocyclic aromatic compounds, in which a furan ring is fused with a coumarin skeleton. Basically there are two types of furocoumarins - angular and linear furocoumarins - depending on the position of the furan ring [1,2].

Furocoumarins have already been reported to exert several beneficial pharmacological effects, like antifungal, antibacterial activites [3]. Some of them were shown to have analgesic and antiinflammatory effects [4], some others have been shown some interesting enzyme inhibitory effects [5]. In spite of these effects, furocoumarins can be dangerous/unsafe. In the literature many of their harmful effect have already been demonstrated such as photosensitizing effect [6].

Coumarins and furocoumarins are found in large quantities in citrus species. The peel contains higher concentrations of the furocoumarins than the pulp.

In my research work different coumarins and furocoumarins were isolated from Citrus trifoliata L. by various separation techniques such as flash chromatography, high performance liquid chromatography and rotation planar chromatography. Six coumarins and furocoumarins auraptene, triphasiol, imperatorin, myrsellin, phellopterin, 7-methoxyscopoletin - were identified by NMR spectroscopy.

The antiproliferative and cytotoxic activity of the isolated compounds were tested in vitro. The bioactivities of certain compounds were examined for the first time. The presented results contribute to a better understanding of risks and beneficial effects of $C$. trifoliata secondary metabolites.

\section{Supervisor: Dezső Csupor}

\section{References}

[1] Chen, C., et al. J. Am. Soc. Hortic. Sci. 2011; 136:358-363.

[2] Melough, M. M, et al. FOOD Chem. Toxicol. 2018;113: 99-107.

[3] Santana, L., et al. Curr. Med. Chem. 2004;11:3239-3261.

[4] Chen, Y. F., et al. Planta Med. 1995;61: 2-8.

[5] Sardari, S., et al. Pharmazie 1999;54: 554-556.

[6] Melough, M. M. \& Chun, O. K. FOOD Chem. Toxicol. 2018;122: 163-171.

[7] Dugrand-Judek, A., et al. PLoS One 2015;10. 